\title{
On Some Numerical Integration Formulas on the $d$-Dimensional Simplex
}

\author{
Filomena Di Tommaso@ and Benaissa Zerroudi
}

\begin{abstract}
In this paper, we consider the problem of the approximation of the integral of a function $f$ over a $d$-dimensional simplex $S$ of $\mathbb{R}^{d}$ by some quadrature formulas which use only the functional and derivative values of $f$ on the boundary of the simplex $S$ or function data at the vertices of $S$, at points on its facets and at its center of gravity. The quadrature formulas are computed by integrating over $S$ a polynomial approximant of $f$ which uses functional and derivative values at the vertices of $S$.
\end{abstract}

Mathematics Subject Classification. Primary 65D32, Secondary 65D30.

Keywords. Numerical integration, Cubature, Simplex.

\section{Introduction}

The problem of the determination of quadrature rules for triangles, tetrahedra and, in general, for $d$-dimensional simplicial domains has reached the attention of a number of scholars starting from the middle of the nineteenth century up today (see [13] and the references therein). Although many papers focus on quadrature rules for triangles $[3,10,12,16]$, only a limited literature is available on the integration in three or higher dimensions $[4,14,17]$. In this paper, we approach the problem of integration over general $d$-dimensional simplices by special type integration formulas which use functional and derivative values of the integrand function $f$ mainly on points on the boundary of the $d$-dimensional simplex $S$. When the nodes lie only on the boundary of $S$ these formulas are called boundary type quadrature formulas and are used when the values of $f$ and its derivatives inside the simplex are not given or are not easily determinable. Applications of these formulas can be realized

This research has been accomplished within the RITA "Research ITalian network on Approximation". This research was supported by GNCS-INdAM 2019 project. The authors would like to thank Professor F. Dell'Accio, Professor O. Nouisser and the anonymous referee for their valuable comments. 
in the framework of the numerical solution of boundary value problems of partial differential equations (see [9] and the references therein).

To reach our goal, we follow the approach proposed in Refs. [1,2]. More precisely, we approximate the integrand function $f$ with a polynomial interpolant $L_{r}^{S}[f](\boldsymbol{x})$ which uses functional and derivative data values up to a fixed order $r \in \mathbb{N}$ at the vertices of $S$, i.e.

$$
f(\boldsymbol{x})=L_{r}^{S}[f](\boldsymbol{x})+R_{r}^{S}[f](\boldsymbol{x}), \quad \boldsymbol{x} \in S,
$$

and then, we integrate both sides of (1.1) over the $d$-dimensional simplex $S$ to get the quadrature formula

$$
\int_{S} f(\boldsymbol{x}) \mathrm{d} \boldsymbol{x}=Q_{r}^{S}[f]+E_{r}^{S}[f]
$$

where

$$
Q_{r}^{S}[f]=\int_{S} L_{r}^{S}[f](\boldsymbol{x}) \mathrm{d} \boldsymbol{x} \quad \text { and } \quad E_{r}^{S}[f]=\int_{S} R_{r}^{S}[f](\boldsymbol{x}) \mathrm{d} \boldsymbol{x} .
$$

The obtained quadrature formula (1.2) uses function and derivative data up to the order $r$ at the vertices of $S$ and has degree of exactness $1+r$, i.e. $E_{r}^{S}[f]=0$ whenever $f$ is a polynomial in $d$ variables of total degree at most $1+r$. The main feature of the quadrature formula $Q_{r}^{S}[f]$ is that it relies only on function and derivative data up to the order $r$ at the vertices of $S$. This motivates us to look for approximations of those derivatives to obtain quadrature formulas which do not use any derivative data. To this end, we restrict to the case $r=1$ and, by following the technique described in Ref. [6], we approximate the first order derivative data by three-point finite differences approximation. According to the choice of the approximation of the derivative data, we get different quadrature formulas with degree of exactness 2 and, to increase the degree of exactness of such formulas, we consider the convex combination of two of them to get a quadrature formula with degree of exactness 3 (see Sect. 3). Finally, we restrict to the two-dimensional case (see Sect. 4) and we provide numerical results to test the approximation accuracies of the proposed formulas (see Sect. 5).

\section{A Quadrature Formula on the $d$-Dimensional Simplex}

\subsection{Preliminaries and Notations}

Let $S \subset \mathbb{R}^{d}$ be a not degenerated $d$-dimensional simplex with vertices $\boldsymbol{v}_{0}, \ldots$, $\boldsymbol{v}_{d} \in \mathbb{R}^{d}$ and

$$
A(S)=\left|\begin{array}{cc}
1 & \boldsymbol{v}_{0} \\
1 & \boldsymbol{v}_{1} \\
1 & \vdots \\
1 & \boldsymbol{v}_{d}
\end{array}\right|
$$

the signed volume of the hyperparallelepiped with vertices $\boldsymbol{v}_{0}, \ldots, \boldsymbol{v}_{d}$. For a point $\boldsymbol{x} \in S$ and for each $l=0,1, \ldots, d$ we denote by $S_{l}(\boldsymbol{x})$ the $d$-dimensional 
simplex of vertices $\boldsymbol{v}_{0}, \ldots, \boldsymbol{v}_{l-1}, \boldsymbol{x}, \boldsymbol{v}_{l+1}, \ldots, \boldsymbol{v}_{d}$. The barycentric coordinates of $\boldsymbol{x}$ with respect to the simplex $S$ are then defined by

$$
\lambda_{l}(\boldsymbol{x})=\frac{A\left(S_{l}\right)}{A(S)}, \quad l=0,1, \ldots, d .
$$

For each $\alpha=\left(\alpha_{1}, \alpha_{2}, \ldots, \alpha_{d}\right) \in \mathbb{N}^{d}$ and $\boldsymbol{x}=\left(x_{1}, \ldots, x_{d}\right) \in \mathbb{R}^{d}$, as usual, we denote by

$$
|\alpha|=\alpha_{1}+\alpha_{2}+\cdots+\alpha_{d}, \quad \alpha !=\alpha_{1} ! \ldots \alpha_{d} !, \quad \boldsymbol{x}^{\alpha}=x_{1}^{\alpha_{1}} x_{2}^{\alpha_{2}} \ldots x_{d}^{\alpha_{d}} .
$$

We also set $\boldsymbol{\lambda}=\left(\lambda_{0}, \lambda_{1}, \lambda_{2}, \ldots, \lambda_{d}\right)$ and $\boldsymbol{\lambda}_{l}=\left(\lambda_{0}, \ldots, \lambda_{l-1}, \lambda_{l+1}, \ldots, \lambda_{d}\right)$ for each $l=0, \ldots, d$. Moreover, we denote by $D^{\alpha} f=\frac{\partial^{|\alpha|} f}{\partial x_{1}^{\alpha_{1}} \partial x_{2}^{\alpha_{2}} \ldots \partial x_{d}^{\alpha_{d}}}$ and by

$$
D_{\boldsymbol{x}-\boldsymbol{v}}^{k} f=\sum_{\substack{|\alpha|=k \\ \alpha \in \mathbb{N}^{d}}} \frac{k !}{\alpha !}(\boldsymbol{x}-\boldsymbol{v})^{\alpha} D^{\alpha} f
$$

the $k$-th order directional derivative of $f$ along the line segment between $\boldsymbol{x}$ and $\boldsymbol{v}$. Finally, we use the notations

$$
D_{i j} f=\left(\boldsymbol{v}_{i}-\boldsymbol{v}_{j}\right) \cdot \nabla f, \quad i, j=0,1, \ldots, d
$$

for the derivative of $f$ along the directed line segment from $\boldsymbol{x}_{j}$ to $\boldsymbol{x}_{i}$ (as usual, - denotes the dot product) and

$$
D_{l}^{\alpha}=D_{0, l}^{\alpha_{1}} D_{1, l}^{\alpha_{2}} \ldots D_{l-1, l}^{\alpha_{l}} D_{l+1, l}^{\alpha_{l+1}} \ldots D_{d, l}^{\alpha_{d}}, \quad l=0,1, \ldots, d,
$$

for the composition of derivatives along the directed sides of the simplex. Under these assumptions, we get the following result.

Lemma 2.1. Let $f \in C^{r}(S)$, then

$$
D_{\boldsymbol{x}-\boldsymbol{v}_{l}}^{k} f\left(\boldsymbol{v}_{l}\right)=\sum_{\substack{|\alpha|=k \\ \alpha \in \mathbb{N}^{d}}} \frac{k !}{\alpha !} D_{l}^{\alpha} f\left(\boldsymbol{v}_{l}\right) \boldsymbol{\lambda}_{l}^{\alpha}(\boldsymbol{x}), \quad l=0,1, \ldots, d,
$$

for any $k \in \mathbb{N}, k \leq r$ and $\boldsymbol{x} \in \mathbb{R}^{d}$.

Proof. Due to the properties satisfied by the barycentric coordinates, for any $l=0,1, \ldots, d$, we have

$$
\begin{aligned}
\boldsymbol{x}-\boldsymbol{v}_{l} & =\boldsymbol{v}_{l} \lambda_{l}(\boldsymbol{x})-\boldsymbol{v}_{l}+\sum_{\substack{j=0 \\
j \neq l}}^{d} \boldsymbol{v}_{j} \lambda_{j}(\boldsymbol{x}) \\
& =\boldsymbol{v}_{l}\left(\lambda_{l}(\boldsymbol{x})-1\right)+\sum_{\substack{j=0 \\
j \neq l}}^{d} \boldsymbol{v}_{j} \lambda_{j}(\boldsymbol{x}) \\
& =-\boldsymbol{v}_{l}\left(\sum_{\substack{j=0 \\
j \neq l}}^{d} \lambda_{j}(\boldsymbol{x})\right)+\sum_{\substack{j=0 \\
j \neq l}}^{d} \boldsymbol{v}_{j} \lambda_{j}(\boldsymbol{x})
\end{aligned}
$$




$$
\begin{aligned}
& =\sum_{\substack{j=0 \\
j \neq l}}^{d}\left(\boldsymbol{v}_{j}-\boldsymbol{v}_{l}\right) \lambda_{j}(\boldsymbol{x}) \\
& =\left(\boldsymbol{v}_{0}-\boldsymbol{v}_{l}, \ldots, \boldsymbol{v}_{l-1}-\boldsymbol{v}_{l}, \boldsymbol{v}_{l+1}-\boldsymbol{v}_{l}, \ldots, \boldsymbol{v}_{d}-\boldsymbol{v}_{l}\right) \cdot \lambda_{l}(\boldsymbol{x})
\end{aligned}
$$

By substituting (2.6) in (2.2) and by definition (2.4), we have

$$
\begin{aligned}
D_{x-v_{l}}^{k} f\left(\boldsymbol{v}_{l}\right) & =\sum_{\substack{|\alpha|=k \\
\alpha \in \mathbb{N}^{d}}} \frac{k !}{\alpha !}\left(\left(\boldsymbol{v}_{0}-\boldsymbol{v}_{l}, \ldots, \boldsymbol{v}_{l-1}-\boldsymbol{v}_{l}, \boldsymbol{v}_{l+1}-\boldsymbol{v}_{l}, \ldots, \boldsymbol{v}_{d}-\boldsymbol{v}_{l}\right) \cdot \boldsymbol{\lambda}_{l}(\boldsymbol{x})\right)^{\alpha} D^{\alpha} f\left(\boldsymbol{v}_{l}\right) \\
& =\sum_{\substack{|\alpha|=k \\
\alpha \in \mathbb{N}^{d}}} \frac{k !}{\alpha !}\left(\left(\boldsymbol{v}_{1}-\boldsymbol{v}_{l}, \ldots, \boldsymbol{v}_{l-1}-\boldsymbol{v}_{l}, \boldsymbol{v}_{l+1}-\boldsymbol{v}_{l}, \ldots, \boldsymbol{v}_{d}-\boldsymbol{v}_{l}\right) D f\left(\boldsymbol{v}_{l}\right)\right)^{\alpha} \boldsymbol{\lambda}_{l}(\boldsymbol{x})^{\alpha} \\
& =\sum_{\substack{|\alpha|=k \\
\alpha \in \mathbb{N}^{d}}} \frac{k !}{\alpha !} D_{l}^{\alpha} f\left(\boldsymbol{v}_{l}\right)^{\alpha} \boldsymbol{\lambda}_{l}(\boldsymbol{x})^{\alpha} .
\end{aligned}
$$

Proposition 2.2. Let $S \subset \mathbb{R}^{d}$ be a not degenerated d-dimensional simplex with vertices $\boldsymbol{v}_{0}, \ldots, \boldsymbol{v}_{d}$ then

$$
\int_{S} \boldsymbol{\lambda}^{\alpha}(\boldsymbol{x}) \mathrm{d} \boldsymbol{x}=\frac{A(S) \alpha !}{(d+|\alpha|) !}, \quad \alpha \in \mathbb{N}^{d+1} .
$$

Proof. See [15, Theorem 2.2].

Proposition 2.3. Let $S \subset \mathbb{R}^{d}$ be a not degenerated d-dimensional simplex with vertices $\boldsymbol{v}_{0}, \boldsymbol{v}_{1}, \ldots, \boldsymbol{v}_{d}$. For any $\boldsymbol{x} \in S$ and $r \in \mathbb{N}$, we have

$$
\int_{S} \sum_{i=0}^{d}\left\|\boldsymbol{x}-\boldsymbol{v}_{i}\right\|^{r+2} \lambda_{i}(\boldsymbol{x}) d \boldsymbol{x} \leq \frac{A(S)}{(d+2) !} \sum_{i=0}^{d} \sum_{j=0}^{d}\left\|\boldsymbol{v}_{i}-\boldsymbol{v}_{j}\right\|^{r+2} .
$$

Proof. By the equality (2.6) and by recalling that $0 \leq \lambda_{j}(\boldsymbol{x}) \leq 1$ for each $\boldsymbol{x} \in S$, we easily obtain

$$
\left\|\boldsymbol{x}-\boldsymbol{v}_{l}\right\|^{r+2} \leq\left(\sum_{j=0}^{d}\left\|\boldsymbol{v}_{j}-\boldsymbol{v}_{l}\right\| \lambda_{j}(\boldsymbol{x})\right)^{r+2} \leq \sum_{j=0}^{d}\left\|\boldsymbol{v}_{j}-\boldsymbol{v}_{l}\right\|^{r+2} \lambda_{j}(\boldsymbol{x})
$$

for any $l=0, \ldots, d$. Consequently,

$$
\begin{aligned}
\int_{S} \sum_{i=0}^{d}\left\|\boldsymbol{x}-\boldsymbol{v}_{i}\right\|^{r+2} \lambda_{i}(\boldsymbol{x}) \mathrm{d} \boldsymbol{x} & \leq \int_{S} \sum_{i=0}^{d} \sum_{j=0}^{d}\left\|\boldsymbol{v}_{j}-\boldsymbol{v}_{i}\right\|^{r+2} \lambda_{j}(\boldsymbol{x}) \lambda_{i}(\boldsymbol{x}) \mathrm{d} \boldsymbol{x} \\
& \leq \sum_{i=0}^{d} \sum_{j=0}^{d}\left\|\boldsymbol{v}_{j}-\boldsymbol{v}_{i}\right\|^{r+2} \int_{S} \lambda_{j}(\boldsymbol{x}) \lambda_{i}(\boldsymbol{x}) \mathrm{d} \boldsymbol{x}
\end{aligned}
$$

and, by Proposition 2.2, we easily get the inequality (2.8). 


\subsection{Construction of the Quadrature Formula}

The multivariate Lagrange interpolation polynomial on the simplex $S$ in barycentric coordinates is

$$
L^{S}[f](\boldsymbol{x})=\sum_{l=0}^{d} \lambda_{l}(\boldsymbol{x}) f\left(\boldsymbol{v}_{l}\right) .
$$

The operator $L^{S}$ reproduces polynomials up to the degree 1 and interpolates the values of $f$ at the vertices $\boldsymbol{v}_{l}$ of the simplex $S$. If the function $f$ belongs to $C^{r}(S)$, we can replace the values $f\left(\boldsymbol{v}_{l}\right)$ by the modified Taylor polynomial of degree $r$ at $\boldsymbol{v}_{\ell}$ proposed in [6], the resulting polynomial operator is

$$
L_{r}^{S}[f](\boldsymbol{x})=\sum_{l=0}^{d}\left(\sum_{k=0}^{r} \frac{a_{r k}}{k !} D_{x-\boldsymbol{v}_{l}}^{k} f\left(\boldsymbol{v}_{l}\right)\right) \lambda_{l}(\boldsymbol{x}), \quad \boldsymbol{x} \in \mathbb{R}^{d},
$$

where $a_{r k}=\frac{(1+r-k) ! r !}{(1+r) !(r-k) !}$. As specified in [6], the operator $L_{r}^{S}[f](\boldsymbol{x})$ reproduces polynomials up to the degree $\max \{1,1+r\}=1+r$. Moreover, for each $\boldsymbol{x} \in S$ and $f \in C^{r+2}(S)$, its remainder term $R_{r}^{S}[f](\boldsymbol{x})=f(\boldsymbol{x})-L_{r}^{S}[f](\boldsymbol{x})$ can be explicitly represented as

$$
R_{r}^{S}[f](\boldsymbol{x})=\sum_{l=0}^{d} \lambda_{t}(x) \int_{0}^{1} \frac{-t(1-t)^{r}}{(1+r) !} D_{x-\boldsymbol{v}_{l}}^{r+2} f\left(\boldsymbol{v}_{l}+t\left(\boldsymbol{x}-\boldsymbol{v}_{l}\right)\right) \mathrm{d} t .
$$

Remark 2.4. Since $S$ is a compact convex domain and $L^{S}$ is a linear bounded operator, in line with [8], $L_{r}^{S}$ can be interpreted as

$$
L_{r}^{S}[f](\boldsymbol{x})=L^{S}\left[\sum_{k=0}^{r} \frac{a_{r k}}{k !} D_{\boldsymbol{x}-.}^{k}\right](\boldsymbol{x})
$$

and, from [8, Proposition 3.4], it follows that $L_{r}^{S}$ inherits the interpolation properties of the Lagrange operator (2.9).

To obtain the desired quadrature formula, we rearrange polynomial (2.10) by taking into account Lemma 2.1. More precisely,

$$
\begin{aligned}
L_{r}^{S}[f](\boldsymbol{x}) & =\sum_{l=0}^{d}\left(\sum_{k=0}^{r} \frac{a_{r k}}{k !} \sum_{\substack{|\alpha|=k \\
\alpha \in \mathbb{N}^{d}}} \frac{k !}{\alpha !} D_{l}^{\alpha} f\left(\boldsymbol{v}_{l}\right) \boldsymbol{\lambda}_{l}^{\alpha}(\boldsymbol{x})\right) \lambda_{l}(\boldsymbol{x}) \\
& =\sum_{l=0}^{d}\left(\sum_{k=0}^{r} \frac{(1+r-k) ! r !}{(1+r) !(r-k) !} \sum_{\substack{|\alpha|=k \\
\alpha \in \mathbb{N}^{d}}} \frac{1}{\alpha !} D_{l}^{\alpha} f\left(\boldsymbol{v}_{l}\right) \boldsymbol{\lambda}_{l}^{\alpha}(\boldsymbol{x})\right) \lambda_{l}(\boldsymbol{x}) \\
& =\sum_{l=0}^{d}\left(\sum_{k=0}^{r} \frac{1+r-k}{1+r} \sum_{\substack{|\alpha|=k \\
\alpha \in \mathbb{N}^{d}}} \frac{1}{\alpha !} D_{l}^{\alpha} f\left(\boldsymbol{v}_{l}\right) \boldsymbol{\lambda}_{l}^{\alpha}(\boldsymbol{x})\right) \lambda_{l}(\boldsymbol{x})
\end{aligned}
$$


and, by the change of dummy index, we get

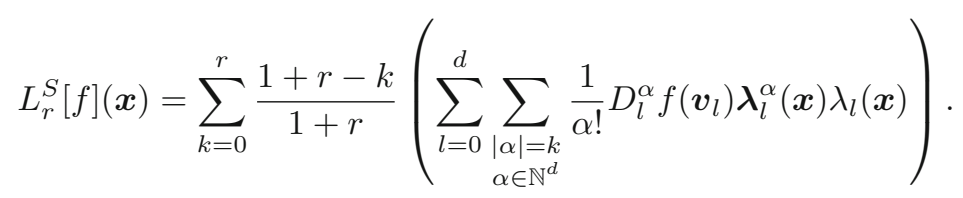

The quadrature formula is then computed by integrating the right hand side of (2.13) on the simplex $S$.

Theorem 2.5. Let $f \in C^{r+2}(S)$. Then

$$
\int_{S} f(\boldsymbol{x}) d \boldsymbol{x}=Q_{r}^{S}[f]+E_{r}^{S}[f],
$$

where

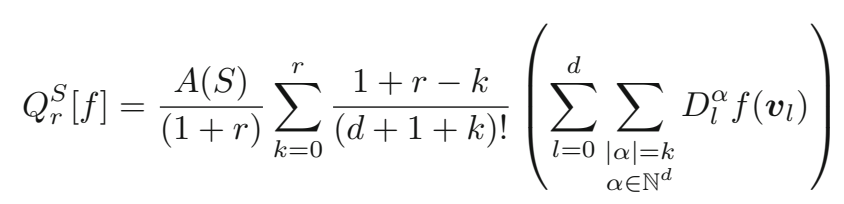

and

$$
\begin{aligned}
E_{r}^{S}[f] & =\int_{S} R_{d}^{S}[f](\boldsymbol{x}) \\
& =\int_{S} \sum_{l=0}^{d}\left(\int_{0}^{1} \frac{-t(1-t)^{r}}{(1+r) !} D_{\boldsymbol{x}-\boldsymbol{v}_{l}}^{r+2} f\left(\boldsymbol{v}_{l}+t\left(\boldsymbol{x}-\boldsymbol{v}_{l}\right)\right) d t\right) \lambda_{l}(\boldsymbol{x}) d \boldsymbol{x} .
\end{aligned}
$$

Moreover, the quadrature formula $Q_{r}^{S}[f]$ has degree of exactness $1+r$.

Proof. By integrating the right hand side of equality (2.13), we get

$$
\begin{aligned}
Q_{r}^{S}[f](\boldsymbol{x}) & =\int_{S} L_{d}^{S}[f](\boldsymbol{x}) \mathrm{d} \boldsymbol{x} \\
& =\sum_{k=0}^{r} \frac{1+r-k}{(1+r)}\left(\sum_{\substack { l=0 \\
\begin{subarray}{c}{|\alpha|=k \\
\alpha \in \mathbb{N}^{d}{ l = 0 \\
\begin{subarray} { c } { | \alpha | = k \\
\alpha \in \mathbb { N } ^ { d } } }\end{subarray}} \frac{1}{\alpha !} D_{l}^{\alpha} f\left(\boldsymbol{v}_{l}\right) \int_{S} \boldsymbol{\lambda}_{l}^{\alpha}(\boldsymbol{x}) \lambda_{l}(\boldsymbol{x}) \mathrm{d} \boldsymbol{x}\right) .
\end{aligned}
$$

By Proposition (2.2)

$$
\int_{S} \boldsymbol{\lambda}_{l}^{\alpha}(\boldsymbol{x}) \lambda_{l}(\boldsymbol{x}) \mathrm{d} \boldsymbol{x}=\frac{A(S) \alpha !}{(d+1+|\alpha|) !}
$$

and then (2.16) becomes

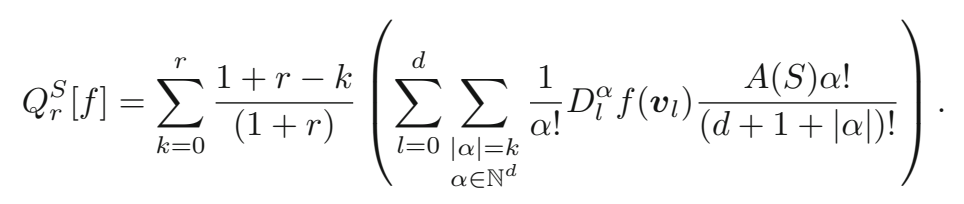


The expression of $E_{r}^{S}[f]$ is obtained by integrating on the simplex $S$ the remainder term $R_{r}^{S}[f](\boldsymbol{x})$ in formula (2.11). Since $R_{r}^{S}[f](\boldsymbol{x})$ vanishes whenever $f$ is a polynomial in $d$ variables of total degree at most $1+r, E_{r}^{S}[f]$ inherits this property and the quadrature formula has degree of exactness $1+r$.

\subsection{Error Bounds}

To give a bound for the remainder term $E_{r}^{S}[f]$ of the quadrature formula in Theorem 2.5, we need some additional notations. More precisely, for a $k$-times continuous differentiable function $f: S \rightarrow \mathbb{R}$, we introduce the norm

$$
\left|D^{k} f\right|_{S}:=\sup _{x \in S} \sup \left\{\left|D_{y}^{k} f(\boldsymbol{x})\right|: \boldsymbol{y} \in \mathbb{R}^{d},\|\boldsymbol{y}\|=1\right\} .
$$

where $\|\cdot\|$ denotes the Euclidean norm in $\mathbb{R}^{d}$, and $\boldsymbol{y}$ is assumed to be a column vector. Consequently, for any $\boldsymbol{x} \in S$ and $\boldsymbol{y} \in \mathbb{R}^{d}$, we have

$$
\left|D_{\boldsymbol{y}}^{k} f(\boldsymbol{x})\right| \leq\left|D^{k} f\right|_{S} \cdot\|\boldsymbol{y}\|^{r+2}
$$

Proposition 2.6. Let $S \subset \mathbb{R}^{d}$ be a not degenerated d-dimensional simplex with vertices $\boldsymbol{v}_{0}, \ldots, \boldsymbol{v}_{d}$ and $f \in C^{r+2}(S)$. Then

$$
\left|E_{r}^{S}[f]\right| \leq \frac{\left|D^{r+2} f\right|_{S}}{(r+2) !(r+1)} \frac{A(S)}{(d+2) !} \sum_{l=0}^{d} \sum_{j=0}^{d}\left\|\boldsymbol{v}_{l}-\boldsymbol{v}_{j}\right\|^{r+2} .
$$

Proof. By taking the modulus of both sides of equality (2.15), by applying the triangular inequality and by bounding the directional derivative of $f$ of order $r+2$ by (2.19), we have

$$
\begin{aligned}
\left|E_{r}^{S}[f]\right| & \leq\left|\int_{S} \sum_{l=0}^{d}\left(\int_{0}^{1} \frac{-t(1-t)^{r}}{(1+r) !} D_{x-\boldsymbol{v}_{l}}^{r+2} f\left(\boldsymbol{v}_{l}+t\left(\boldsymbol{x}-\boldsymbol{v}_{l}\right)\right) \mathrm{d} t\right) \lambda_{l}(\boldsymbol{x}) \mathrm{d} \boldsymbol{x}\right| \\
& \leq\left|D^{r+2} f\right|_{S} \int_{S} \sum_{l=0}^{d}\left(\int_{0}^{1} \frac{t(1-t)^{r}}{(1+r) !}\left\|\boldsymbol{x}-\boldsymbol{v}_{l}\right\|^{r+2} \mathrm{~d} t\right) \lambda_{l}(\boldsymbol{x}) \mathrm{d} \boldsymbol{x} \\
& \leq \frac{\left|D^{r+2} f\right|_{S}}{(1+r) !} \int_{S} \sum_{l=0}^{d}\left\|\boldsymbol{x}-\boldsymbol{v}_{l}\right\|^{r+2}\left(\int_{0}^{1} t(1-t)^{r} \mathrm{~d} t\right) \lambda_{l}(\boldsymbol{x}) \mathrm{d} \boldsymbol{x} .
\end{aligned}
$$

Using the inequality in Proposition (2.3), and by the fact that

$$
\int_{0}^{1} t(1-t)^{r} \mathrm{~d} t=\frac{1}{(r+2)(r+1)}
$$

we have

$$
\left|E_{r}^{S}[f]\right| \leq \frac{\left|D^{r+2} f\right|_{S}}{(1+r) !(r+1)(r+2)} \frac{A(S)}{(d+2) !} \sum_{l=0}^{d} \sum_{j=0}^{d}\left\|\boldsymbol{v}_{l}-\boldsymbol{v}_{j}\right\|^{r+2}
$$

and then (2.20).

Remark 2.7. It is worth noting that Theorem 2.5 gives a quadrature formula obtained by integrating both sides of the expression in [6, Theorem 1] and the bound in Proposition 2.6 is nothing but the integral of the bound given in [6, Theorem 2], where $\Omega=S, m=1$ and $\phi_{i}(\boldsymbol{x})=\lambda_{i}(\boldsymbol{x})$. Consequently, the bound (2.20) is the best possible estimation for each $r \in \mathbb{N}_{0}$. 


\section{Integration Formulas on the Simplex with Only Function Data}

The main feature of the quadrature formula (2.14) is that it uses only derivatives of $f$ along the edges of $S$; this motivates to consider approximations of those derivatives to obtain quadrature formulas which use the function data at the vertices of the simplex $S$, at points on its facets or at its center of gravity. To this aim, we focus on the case $r=1$ and we consider different kinds of approximation of the derivatives in (2.14). For $r=1$, the quadrature formula (2.14) in Theorem 2.5 becomes

$$
Q_{1}^{S}[f]=\frac{A(S)}{(d+1) !} \sum_{l=0}^{d} f\left(\boldsymbol{v}_{l}\right)+\frac{A(S)}{2(d+2) !} \sum_{l=0}^{d} \sum_{\substack{|\alpha|=1 \\ \alpha \in \mathbb{N}^{d}}} D_{l}^{\alpha} f\left(\boldsymbol{v}_{l}\right) .
$$

Proposition 3.1. Let $f: S \rightarrow \mathbb{R}$ be a 3-times continuous differentiable function on $S$, then

$$
\begin{aligned}
\tilde{Q}_{1}^{S}[f]= & \frac{A(S)}{(d+2) !}\left((3 d+2) \sum_{l=0}^{d} f\left(\boldsymbol{v}_{l}\right)-4 \sum_{l=0}^{d-1} \sum_{r=l+1}^{d} f\left(\frac{\boldsymbol{v}_{r}+\boldsymbol{v}_{l}}{2}\right)\right) \\
& +\frac{A(S)}{2(d+2) !} \sum_{l=0}^{d-1} \sum_{r=l+1}^{d} \varepsilon_{l, r} .
\end{aligned}
$$

where

$$
\begin{aligned}
\varepsilon_{l, r}[f]= & D_{\boldsymbol{v}_{l}-\boldsymbol{v}_{r}}^{3} f\left(\boldsymbol{v}_{r}+\xi_{1}\left(\boldsymbol{v}_{l}-\boldsymbol{v}_{r}\right)\right) \\
& -D_{\boldsymbol{v}_{l}-\boldsymbol{v}_{r}}^{3} f\left(\boldsymbol{v}_{r}+\xi_{2}\left(\boldsymbol{v}_{l}-\boldsymbol{v}_{r}\right)\right), \quad \xi_{1}, \xi_{2} \in[0,1] .
\end{aligned}
$$

Moreover, the quadrature formula (3.2) has degree of exactness 2.

Proof. By definition (2.4), the sum of first-order derivatives in the second term of $Q_{1}^{S}[f]$ can be rewritten as

$$
\sum_{l=0}^{d} \sum_{\substack{\alpha \mid=1 \\ \alpha \in \mathbb{N}^{d}}} D_{l}^{\alpha} f\left(\boldsymbol{v}_{l}\right)=\sum_{l=0}^{d} \sum_{\substack{r=0 \\ r \neq l}}^{d} D_{r l} f\left(\boldsymbol{v}_{l}\right)=\sum_{l=0}^{d-1} \sum_{r=l+1}^{d}\left(D_{l r} f\left(\boldsymbol{v}_{r}\right)-D_{l r} f\left(\boldsymbol{v}_{l}\right)\right),
$$

where the differences of directional derivatives along the edges of the simplex $S$ can be replaced by a three-point finite difference approximation. To do this, let us recall that for a univariate function $g$, it is possible to consider the derivation formula

$$
g^{\prime}(a-h)=\frac{1}{h}\left(-\frac{1}{2} g(a+h)+2 g(a)-\frac{3}{2} g(a-h)\right)+\frac{h^{2}}{3} g^{\prime \prime \prime}(\xi)
$$

for some $\xi \in[a-h, a+h]$. Using this formula with $h= \pm 1 / 2$ and $a=1 / 2$ we get a three-point approximation for $g^{\prime}(0)-g^{\prime}(1)$ with a remainder term 
which is expressed in terms of the modulus of continuity of $g^{\prime \prime \prime}[6$, Section 5.1]. By applying the formula (3.4) along the edges of $S$ we get

$$
D_{l r} f\left(\boldsymbol{v}_{r}\right)-D_{l r} f\left(\boldsymbol{v}_{l}\right)=4\left(f\left(\boldsymbol{v}_{r}\right)-2 f\left(\frac{\boldsymbol{v}_{r}+\boldsymbol{v}_{l}}{2}\right)+f\left(\boldsymbol{v}_{l}\right)\right)+\varepsilon_{l, r}
$$

with

$$
\left|\varepsilon_{l, r}\right| \leq \frac{1}{12} \omega\left(D_{l}^{3} f\left((1-t) \boldsymbol{v}_{l}+t \boldsymbol{v}_{r}\right), 1\right),
$$

where $\omega$ denotes the modulus of continuity with respect to $t \in[0,1]$. By substituting expression (3.5) in (3.3) and by rearranging, we get

$$
\sum_{l=0}^{d} \sum_{\substack{\alpha \mid=1 \\ \alpha \in \mathbb{N}^{d}}} D_{l}^{\alpha} f\left(\boldsymbol{v}_{l}\right)=4 d \sum_{l=0}^{d} f\left(\boldsymbol{v}_{l}\right)-8 \sum_{l=0}^{d-1} \sum_{r=l+1}^{d} f\left(\frac{\boldsymbol{v}_{r}+\boldsymbol{v}_{l}}{2}\right)+\sum_{l=0}^{d-1} \sum_{r=l+1}^{d} \varepsilon_{l, r} .
$$

Finally, by substituting (3.6) in (3.1), we get

$$
\begin{aligned}
\tilde{Q}_{1}^{S}[f]= & \frac{A(S)}{(d+2) !}\left((3 d+2) \sum_{l=0}^{d} f\left(\boldsymbol{v}_{l}\right)-4 \sum_{l=0}^{d-1} \sum_{r=l+1}^{d} f\left(\frac{\boldsymbol{v}_{r}+\boldsymbol{v}_{l}}{2}\right)\right) \\
& +\frac{A(S)}{2(d+2) !} \sum_{l=0}^{d-1} \sum_{r=l+1}^{d} \varepsilon_{l, r} .
\end{aligned}
$$

$\varepsilon_{l, r}[f]=0$ whenever $f$ is a polynomial in $d$ variables of total degree 2 and this implies that $\tilde{Q}_{1}^{S}[f]$ has degree of exactness 2 .

Proposition 3.2. Let $S \subset \mathbb{R}^{d}$ be a not degenerated d-dimensional simplex with vertices $\left(\boldsymbol{v}_{l}\right)_{l=0,1, \ldots, d}$. Let us denote by $s_{l}^{d-1}, l=0,1, \ldots, d$ the facet opposite to the vertex $\boldsymbol{v}_{l}$ and by $\boldsymbol{g}_{l}$ the barycenter of $s_{l}^{d-1}$. For all $\alpha \in(0,1)$ we have

$$
\begin{gathered}
\int_{S} f(\boldsymbol{x}) \mathrm{d} \boldsymbol{x}=\frac{A(S)}{2(d+2) !}\left(\frac{\alpha(d+4)-d}{\alpha} \sum_{l=0}^{d} f\left(\boldsymbol{v}_{l}\right)+\frac{d}{\alpha-\alpha^{2}} \sum_{l=0}^{d} f\left(\boldsymbol{y}_{l}(\alpha)\right)\right. \\
\left.+\frac{\alpha d}{\alpha-1} \sum_{l=0}^{d} f\left(\boldsymbol{g}_{l}\right)\right)+R(d, \alpha)[f]
\end{gathered}
$$

with $\boldsymbol{y}_{l}(\alpha)=\boldsymbol{v}_{l}+\alpha\left(\boldsymbol{g}_{l}-\boldsymbol{v}_{l}\right)$ and

$$
\begin{aligned}
R(d, \alpha)[f]= & \frac{d A(S)}{4(d+2) !\left(\alpha-\alpha^{2}\right)} \sum_{l=0}^{d}\left(\alpha^{2} \int_{0}^{1}(1-t)^{2} D_{\boldsymbol{g}_{l}-\boldsymbol{v}_{l}}^{3} f\left(\boldsymbol{v}_{l}+t\left(\boldsymbol{g}_{l}-\boldsymbol{v}_{l}\right)\right) \mathrm{d} t\right. \\
& \left.-\int_{0}^{\alpha}(\alpha-t)^{2} D_{\boldsymbol{g}_{l}-\boldsymbol{v}_{l}}^{3} f\left(\boldsymbol{v}_{l}+t\left(\boldsymbol{g}_{l}-\boldsymbol{v}_{l}\right)\right) \mathrm{d} t\right) .
\end{aligned}
$$

The quadrature formula (3.7) has degree of exactness 2. 
Proof. Let $\boldsymbol{g}_{l}$ be the barycenter of $s_{l}^{d-1}$, by Lemma (2.1) the sum of first-order derivatives along the edges of $S$ in (3.1) can be rewritten as

$$
\sum_{\substack{l=0 \\|\alpha|=1 \\ \alpha \in \mathbb{N}^{d}}}^{d} D_{l}^{\alpha} f\left(\boldsymbol{v}_{l}\right) \boldsymbol{\lambda}^{\alpha}\left(\boldsymbol{g}_{l}\right)=\sum_{l=0}^{d} D_{\boldsymbol{g}_{l}-\boldsymbol{v}_{l}} f\left(\boldsymbol{v}_{l}\right)
$$

and, since $\lambda_{k}\left(\boldsymbol{g}_{l}\right)=\frac{1}{d}$, for each $l, k=0, \ldots, d$, then

$$
\sum_{l=0}^{d} \sum_{\substack{|\alpha|=1 \\ \alpha \in \mathbb{N}^{d}}} D_{l}^{\alpha} f\left(\boldsymbol{v}_{l}\right)=d \sum_{l=0}^{d} D_{\boldsymbol{g}_{l}-\boldsymbol{v}_{l}} f\left(\boldsymbol{v}_{l}\right) .
$$

By substituting (3.9) in (3.1), we get

$$
Q_{1}^{S}[f]=\frac{A(S)}{(d+1) !} \sum_{l=0}^{d} f\left(\boldsymbol{v}_{l}\right)+\frac{d A(S)}{2(d+2) !} \sum_{l=0}^{d} D_{g_{l}-\boldsymbol{v}_{l}} f\left(\boldsymbol{v}_{l}\right) .
$$

To have a three point finite difference approximation of the directional derivatives in (3.10), for each $l=0, \ldots, d$, let us introduce the univariate function

$$
h_{l}:\left\{\begin{array}{l}
{[0,1] \rightarrow \mathbb{R},} \\
t \mapsto h_{l}(t)=f\left(\boldsymbol{v}_{l}+t\left(\boldsymbol{g}_{l}-\boldsymbol{v}_{l}\right)\right) .
\end{array}\right.
$$

For $t=1$ and $t=\alpha \in(0,1)$, the second-order Taylor expansion of $h_{l}(1)$ and $h_{l}(\alpha)$ centered at 0 with integral remainder are

$$
h_{l}(1)=h_{l}(0)+h_{l}^{\prime}(0)+\frac{1}{2} h_{l}^{\prime \prime}(0)+\frac{1}{2} \int_{0}^{1}(1-t)^{2} h_{l}^{\prime \prime \prime}(t) \mathrm{d} t
$$

and

$$
h_{l}(\alpha)=h_{l}(0)+\alpha h_{l}^{\prime}(0)+\frac{1}{2} \alpha^{2} h_{l}^{\prime \prime}(0)+\frac{1}{2} \int_{0}^{\alpha}(\alpha-t)^{2} h_{l}^{\prime \prime \prime}(t) \mathrm{d} t .
$$

Then, by (3.12) and (3.13), we get

$$
h_{l}(\alpha)-\alpha^{2} h_{l}(1)=\left(1-\alpha^{2}\right) h_{l}(0)+\left(\alpha-\alpha^{2}\right) h_{l}^{\prime}(0)+R_{l}[f](\alpha)
$$

where

$$
R_{l}[f](\alpha)=\frac{1}{2} \int_{0}^{\alpha}(\alpha-t)^{2} h_{l}^{\prime \prime \prime}(t) \mathrm{d} t-\alpha^{2} \frac{1}{2} \int_{0}^{1}(1-t)^{2} h_{l}^{\prime \prime \prime}(t) \mathrm{d} t .
$$

Therefore, by (3.14) it follows that

$$
h_{l}^{\prime}(0)=\frac{1}{\alpha-\alpha^{2}} h_{l}(\alpha)-\frac{\alpha}{1-\alpha} h_{l}(1)-\frac{1+\alpha}{\alpha} h_{l}(0)+\frac{1}{\alpha-\alpha^{2}} R_{l}(\alpha)[f] .
$$

By rewriting equality (3.15) in terms of $f$ we get

$$
\begin{aligned}
D_{\boldsymbol{g}_{l}-\boldsymbol{v}_{l}} f\left(\boldsymbol{v}_{l}\right)= & \frac{1}{\alpha-\alpha^{2}} f\left(\alpha \boldsymbol{g}_{l}+(1-\alpha) \boldsymbol{v}_{l}\right)-\frac{\alpha}{1-\alpha} f\left(\boldsymbol{g}_{l}\right)-\frac{1+\alpha}{\alpha} f\left(\boldsymbol{v}_{l}\right) \\
& +\frac{1}{\alpha-\alpha^{2}} R_{l}(\alpha)[f]
\end{aligned}
$$


where

$$
\begin{aligned}
R_{l}(\alpha)[f]= & \frac{1}{2}\left(\alpha^{2} \int_{0}^{1}(1-t)^{2} D_{\boldsymbol{g}_{l}-\boldsymbol{v}_{l}}^{3} f\left(\boldsymbol{v}_{l}+t\left(\boldsymbol{g}_{l}-\boldsymbol{v}_{l}\right)\right) \mathrm{d} t\right. \\
& \left.-\int_{0}^{\alpha}(\alpha-t)^{2} D_{\boldsymbol{g}_{l}-\boldsymbol{v}_{l}}^{3} f\left(\boldsymbol{v}_{l}+t\left(\boldsymbol{g}_{l}-\boldsymbol{v}_{l}\right)\right) \mathrm{d} t\right) .
\end{aligned}
$$

Finally, by substituting (3.16) in (3.1), we get (3.7). $R(\alpha)[f]=0$ whenever $f$ is a polynomial in $d$ variables of total degree 2 and, therefore, the quadrature formula (3.7) has degree of exactness 2.

Remark 3.3. 1. For $\alpha=\frac{d}{d+4}$ the formula (3.7) becomes

$$
\begin{aligned}
\int_{S} f(\boldsymbol{x}) \mathrm{d} \boldsymbol{x}= & \frac{A(S)}{2(d+2) !}\left(\frac{(d+4)^{2}}{4} \sum_{l=0}^{d} f\left(\frac{d \boldsymbol{g}_{l}+4 \boldsymbol{v}_{l}}{d+4}\right)-\frac{d^{2}}{4} \sum_{l=0}^{d} f\left(\boldsymbol{g}_{l}\right)\right) \\
& +R\left(d, \frac{d}{d+4}\right)[f],
\end{aligned}
$$

that is a quadrature formula which uses only the function data at the points $\boldsymbol{g}_{l}$ and $\frac{d \boldsymbol{g}_{l}+4 \boldsymbol{v}_{l}}{d+4}, l=0, \ldots, d$, and is exact for all polynomial of degree less than or equal to 2 .

2. For $\alpha=\frac{d}{d+1}$, the quadrature formula (3.7) becomes,

$$
\begin{aligned}
\int_{S} f(\boldsymbol{x}) \mathrm{d} \boldsymbol{x}= & \frac{A(S)}{2(d+2) !}\left(3 \sum_{l=0}^{d} f\left(\boldsymbol{v}_{l}\right)+(d+1)^{3} f\left(\boldsymbol{x}^{*}\right)-d^{2} \sum_{l=0}^{d} f\left(\boldsymbol{g}_{l}\right)\right) \\
& +R\left(d, \frac{d}{d+1}\right)[f]
\end{aligned}
$$

where $\boldsymbol{x}^{*}$ is the center of gravity of $S$.

To improve the approximation accuracy of the quadrature formula (3.19), let us consider a convex combination of this formula with a multivariate Simpson rule for a simplex proposed in [7, Theorem 5.1]. For a particular value of the parameter of the linear combination, we are able to get a quadrature formula with an higher degree of exactness.

Corollary 3.4. Let $f: S \rightarrow \mathbb{R}$ be a 3-times continuous differentiable function on $S$. Let us denote by $\boldsymbol{x}^{*}$ the center of gravity of $S$, by $s_{l}^{d-1}, l=0,1, \ldots, d$ the facets of $S$ opposite to the vertex $\boldsymbol{v}_{l}$ and by $\boldsymbol{g}_{l}$ the barycenter of $s_{l}^{d-1}$. Then,

$$
\int_{S} f(\boldsymbol{x}) d \boldsymbol{x}=\tau F_{1}[f]+(1-\tau) F_{2}[f]+\tilde{R}(\tau)[f], \quad \tau \in \mathbb{R}
$$

where

$$
F_{1}[f]=\frac{A(S)}{d !}\left(\frac{d+1}{d+2} f\left(\boldsymbol{x}^{*}\right)+\frac{1}{(d+1)(d+2)} \sum_{l=0}^{d} f\left(\boldsymbol{v}_{l}\right)\right)
$$


is the multivariate Simpson rule for a simplex [7, Theorem 5.1],

$$
F_{2}[f]=\frac{A(S)}{2(d+2) !}\left(3 \sum_{l=0}^{d} f\left(\boldsymbol{v}_{l}\right)+(d+1)^{3} f\left(\boldsymbol{x}^{*}\right)-d^{2} \sum_{l=0}^{d} f\left(\boldsymbol{g}_{l}\right)\right)
$$

is given by (3.7) for $\alpha=\frac{d}{d+1}$ and

$$
\tilde{R}(\tau)[f]=\tau R_{d}^{S i}[f]+(1-\tau) R\left(d, \frac{d}{d+1}\right)[f]
$$

with $R_{d}^{S i}[f]$ denoting the remainder term in the multivariate Simpson rule for a simplex. For all $\tau \in \mathbb{R}$ we have $\tilde{R}(\tau)[f]=0$, whenever $f$ is a polynomial in $d$ variables of total degree at most 2 .

Proof. Since $R_{d}^{S i}[f]=0$ and $R\left(d, \frac{d}{d+1}\right)[f]=0$ whenever $f$ is a polynomial in $d$ variables of total degree less than or equal to 2 , it easily follows that $\tilde{R}(\tau)[f]$ vanishes for each polynomial of degree at most 2 .

For $\tau=\frac{3(d+1)}{d+3}$, the family of quadrature formulas (3.20) yields to a formula which has degree of exactness 3 .

Theorem 3.5. Let $f: S \rightarrow \mathbb{R}$ be a 3-times continuous differentiable function on $S$ and let us denote by $\boldsymbol{x}^{*}$ the center of gravity of $S$, by $s_{l}^{d-1}, l=0,1, \ldots, d$ the facet opposite to the vertex $\boldsymbol{v}_{l}$ and by $\boldsymbol{g}_{l}$ the barycenter of $s_{l}^{d-1}$. The quadrature formula

$$
\begin{aligned}
\int_{S} f(\boldsymbol{x}) d \boldsymbol{x}= & \frac{A(S)}{(d+3) !}\left(3 \sum_{l=0}^{d} f\left(\boldsymbol{v}_{l}\right)+d^{3} \sum_{l=0}^{d} f\left(\boldsymbol{g}_{l}\right)+(d+1)^{3}(3-d) f\left(\boldsymbol{x}^{*}\right)\right) \\
& +\tilde{R}\left(\frac{3(d+1)}{d+3}\right)[f]
\end{aligned}
$$

with $\tilde{R}\left(\frac{3(d+1)}{d+3}\right)[f]$ defined in (3.21), has degree of exactness 3 .

Proof. For $\tau=\frac{3(d+1)}{d+3}$, the quadrature formula (3.20) reduces to

$$
\begin{aligned}
& \int_{S} f(\boldsymbol{x}) \mathrm{d} \boldsymbol{x} \approx F_{3}[f] \\
& \quad=\frac{A(S)}{(d+3) !}\left(3 \sum_{l=0}^{d} f\left(\boldsymbol{v}_{l}\right)+d^{3} \sum_{l=0}^{d} f\left(\boldsymbol{g}_{l}\right)+(d+1)^{3}(3-d) f\left(\boldsymbol{x}^{*}\right)\right)
\end{aligned}
$$

and, by Corollary 3.4, it follows that $F_{3}[f]$ has degree of exactness 2 . Let $P_{3}(\boldsymbol{x})$ be a polynomial in $d$ variables of degree 3 ; we can write $P_{3}(\boldsymbol{x})$ as

$$
P_{3}(\boldsymbol{x})=P_{2}(\boldsymbol{x})+\sum_{i=0}^{d} c_{i} x_{i}^{3}+\sum_{i=0}^{d} \sum_{\substack{j=0 \\ j \neq i}}^{d} b_{i j} x_{i}^{2} x_{j}+\sum_{i=0}^{d} \sum_{\substack{j=0 \\ j \neq i}}^{d} \sum_{\substack{k=0 \\ k \neq i, j}}^{d} d_{i j k} x_{i} x_{j} x_{k}
$$


where $P_{2}(\boldsymbol{x})$ is a polynomial of degree 2 . Therefore, it is sufficient to prove that $F_{3}[\cdot]$ is exact for the monomials

$$
M_{1}=x_{i}^{3} ; M_{2}=x_{i}^{2} x_{j} \quad(j \neq i) ; M_{3}=x_{i} x_{j} x_{k} \quad(k \neq i, j) \quad i, j, k=0, \ldots, d .
$$

Thanks to the linear isomorphism which maps the standard simplex $\Delta_{d}$ of $\mathbb{R}^{d}$ to a generic simplex $S$, without loss of generality, we can restrict to the case of the simplex $\Delta_{d}$ of vertices $\boldsymbol{v}_{0}=(0, \ldots, 0) ; \boldsymbol{v}_{1}=(1,0, \ldots, 0) ; \boldsymbol{v}_{l}=$ $(0,0, \ldots, 1, \ldots, 0) ; \boldsymbol{v}_{d}=(0, \ldots, 0,1)$. The center of gravity of $\Delta_{d}$ is $\boldsymbol{x}^{*}=$ $\left(\frac{1}{d+1}, \ldots, \frac{1}{d+1}\right)$ and the barycenter of the facets are

$$
\boldsymbol{g}_{0}=\left(\frac{1}{d}, \frac{1}{d}, \ldots, \frac{1}{d}\right), \boldsymbol{g}_{1}=\left(0, \frac{1}{d}, \ldots, \frac{1}{d}\right), \ldots, \boldsymbol{g}_{d}=\left(\frac{1}{d}, \frac{1}{d}, \ldots, 0\right) .
$$

Let us now consider $M_{1}=x_{i}^{3}, i=0, \ldots, d$. The exact integral of $M_{1}$ over the simplex $\Delta_{d}$ is $[11]$

$$
\int_{\Delta_{d}} M_{1} \mathrm{~d} \boldsymbol{x}=\frac{6}{(d+3) !}
$$

and, in addition,

$$
\begin{aligned}
& \sum_{l=0}^{d} f\left(\boldsymbol{v}_{l}\right)=1, \\
& \sum_{l=0}^{d} f\left(\boldsymbol{g}_{l}\right)=\frac{d}{d^{3}}, \\
& f\left(\boldsymbol{x}^{*}\right)=\frac{1}{(d+1)^{3}} .
\end{aligned}
$$

By substituting equalities (3.24) in the quadrature formula (3.23) we have

$$
\begin{aligned}
F_{3}\left[M_{1}\right] & =\frac{A\left(\Delta_{d}\right)}{(d+3) !}\left(3+d^{3} \frac{d}{d^{3}}+(d+1)^{3}(3-d) \frac{1}{(d+1)^{3}}\right) \\
& =\frac{6}{(d+3) !}=\int_{\Delta_{d}} M_{1} \mathrm{~d} \boldsymbol{x} .
\end{aligned}
$$

Let us consider $M_{2}=x_{i}^{3} x_{j}, i, j=0, \ldots, d$ and $j \neq i$. The exact integral of $M_{2}$ over the simplex $\Delta_{d}$ is [11]

$$
\int_{\Delta_{d}} M_{2} \mathrm{~d} \boldsymbol{x}=\frac{2}{(d+3) !},
$$

and, in addition,

$$
\begin{aligned}
& \sum_{l=0}^{d} f\left(\boldsymbol{v}_{l}\right)=0, \\
& \sum_{l=0}^{d} f\left(\boldsymbol{g}_{l}\right)=\frac{d-1}{d^{3}}, \\
& f\left(\boldsymbol{x}^{*}\right)=\frac{1}{(d+1)^{3}} .
\end{aligned}
$$

By substituting equalities (3.25) in the quadrature formula (3.23) we have

$$
F_{3}\left[M_{2}\right]=\frac{A\left(\Delta_{d}\right)}{(d+3) !}\left(0+d^{3} \frac{d-1}{d^{3}}+(d+1)^{3}(3-d) \frac{1}{(d+1)^{3}}\right)
$$




$$
=\frac{2}{(d+3) !}=\int_{\Delta_{d}} M_{2} \mathrm{~d} \boldsymbol{x} .
$$

Finally, let us consider $M_{3}=x_{i} x_{j} x_{k}, i, j, k=0, \ldots, d$ and $k \neq i, j$ for which the exact integral over the simplex $\Delta_{d}$ is [11]

$$
\int_{\Delta_{d}} M_{3} \mathrm{~d} \boldsymbol{x}=\frac{1}{(d+3) !}
$$

and

$$
\begin{aligned}
& \sum_{l=0}^{d} f\left(\boldsymbol{v}_{l}\right)=0 \\
& \sum_{l=0}^{d} f\left(\boldsymbol{g}_{l}\right)=\frac{d-2}{d^{3}}, \\
& f\left(\boldsymbol{x}^{*}\right)=\frac{1}{(d+1)^{3}} .
\end{aligned}
$$

By substituting equalities (3.26) in the quadrature formula (3.23), we have

$$
\begin{aligned}
F_{3}\left[M_{3}\right] & =\frac{A(\tilde{S})}{(d+3) !}\left(0+d^{3} \frac{d-2}{d^{3}}+(d+1)^{3}(3-d) \frac{1}{(d+1)^{3}}\right) \\
& =\frac{1}{(d+3) !}=\int_{\Delta_{d}} M_{3} \mathrm{~d} \boldsymbol{x} .
\end{aligned}
$$

Then

$$
\int_{\Delta_{d}} P_{3}(\boldsymbol{x}) d \boldsymbol{x}=F_{3}\left[P_{3}\right]
$$

and this shows that the degree of exactness of the quadrature formula (3.22) is 3 .

\section{The 2-Simplex Case}

In this section, we restrict to the case $d=2$ in which $S$ is a triangle of vertices $\boldsymbol{v}_{0}, \boldsymbol{v}_{1}, \boldsymbol{v}_{2}$. In this particular case, the quadrature formula (2.14) reduces to

$$
\begin{aligned}
Q_{r}^{S}[f]= & \frac{1}{(1+r)} \sum_{k=0}^{r} \frac{1+r-k}{(3+k) !} \sum_{j=0}^{k}\left(D_{\boldsymbol{v}_{1}-\boldsymbol{v}_{0}}^{j} D_{\boldsymbol{v}_{2}-\boldsymbol{v}_{0}}^{k-j} f\left(\boldsymbol{v}_{0}\right)\right. \\
& +D_{\boldsymbol{v}_{0}-\boldsymbol{v}_{1}}^{j} D_{\boldsymbol{v}_{2}-\boldsymbol{v}_{1}}^{k-j} f\left(\boldsymbol{v}_{1}\right) \\
& \left.+D_{\boldsymbol{v}_{0}-\boldsymbol{v}_{2}}^{j} D_{\boldsymbol{v}_{1}-\boldsymbol{v}_{2}}^{k-j} f\left(\boldsymbol{v}_{2}\right)\right)
\end{aligned}
$$

and, by easy computations, the bound for the approximation error becomes

$$
\left|E_{r}^{\Delta_{2}}[f]\right| \leq \frac{\left|D^{r+2} f\right|_{S}}{12(r+2) !(1+r)}\left(1+\sqrt{2^{r}}\right) .
$$

The quadrature formula (3.19), which has degree of exactness 2 and uses only function data at the vertices of $S$, at the midpoints of its sides and at its center of gravity, becomes

$$
\int_{S} f(\boldsymbol{x}) \mathrm{d} \boldsymbol{x}=\tilde{Q}_{1}^{S}[f]+R\left(2, \frac{2}{3}\right)[f]
$$


where

$$
\tilde{Q}_{2}^{S}[f]=\frac{A(S)}{16}\left(\sum_{l=0}^{2} f\left(\boldsymbol{v}_{l}\right)-\frac{4}{3} \sum_{l=0}^{1} \sum_{r=l+1}^{2} f\left(\boldsymbol{g}_{l}\right)+9 f\left(\boldsymbol{x}^{*}\right)\right)
$$

and

$$
\begin{gathered}
R\left(2, \frac{2}{3}\right)[f]=\frac{3 A(S)}{32} \sum_{l=0}^{2}\left(\frac{4}{9} \int_{0}^{1}(1-t)^{2} D_{\boldsymbol{g}_{l}-\boldsymbol{v}_{l}}^{3} f\left(\boldsymbol{v}_{l}+t\left(\boldsymbol{g}_{l}-\boldsymbol{v}_{l}\right)\right) \mathrm{d} t\right. \\
\left.-\int_{0}^{\frac{2}{3}}\left(\frac{2}{3}-t\right)^{2} D_{\boldsymbol{g}_{l}-\boldsymbol{v}_{l}}^{3} f\left(\boldsymbol{v}_{l}+t\left(\boldsymbol{g}_{l}-\boldsymbol{v}_{l}\right)\right) \mathrm{d} t\right) .
\end{gathered}
$$

The quadrature formula (3.22), which has degree of exactness 3 and uses only function data at the vertices of $S$, at the midpoints of its sides and at its center of gravity, becomes

$$
\tilde{Q}_{3}^{S}[f]=\frac{A(S)}{40}\left(\sum_{l=0}^{d} f\left(\boldsymbol{v}_{l}\right)+\frac{8}{3} \sum_{l=0}^{d} f\left(\boldsymbol{g}_{l}\right)+9 f\left(\boldsymbol{x}^{*}\right)\right),
$$

where $\boldsymbol{g}_{l}=\frac{\boldsymbol{v}_{l}+\boldsymbol{v}_{r}}{2}$, i.e. $\boldsymbol{g}_{l}$ is nothing but the midpoint of the side of the triangle $S$ opposite to $\boldsymbol{v}_{l}$. For $d=2$ and $\alpha=1 / 3$ the formula (3.7), which has degree of exactness 2 and uses function data at the midpoints of the sides of $S$ and at the points $\frac{g_{l}+2 v_{l}}{3}, l=0, \ldots, d$, becomes

$$
\int_{S} f(\boldsymbol{x}) \mathrm{d} \boldsymbol{x}=\widehat{Q}_{2}^{S}[f]+R\left(2, \frac{1}{3}\right)[f]
$$

with

$$
\widehat{Q}_{2}^{S}[f]=\frac{A(S)}{48}\left(9 \sum_{l=0}^{2} f\left(\frac{\boldsymbol{g}_{l}+2 \boldsymbol{v}_{l}}{3}\right)-\sum_{l=0}^{2} f\left(\boldsymbol{g}_{l}\right)\right)
$$

and $R\left(2, \frac{1}{3}\right)[f]$ defined in $(3.8)$.

To enhance the degree of exactness of the quadrature formula (4.6), let us consider the midpoint formula for the 2-dimensional simplex [5]

$$
\int_{S} f(\boldsymbol{x}) \mathrm{d} \boldsymbol{x}=\frac{A(S)}{6} \sum_{r=0}^{2} f\left(\boldsymbol{g}_{r}\right)+E[f]
$$

where $E[f]=0$ whenever $f$ is a polynomial in 2 variables of total degree at most 2. We set

$$
Q_{2}^{\text {mid }}[f]=\frac{A(S)}{6} \sum_{l=0}^{2} f\left(\boldsymbol{g}_{l}\right)
$$

and define the convex combination

$$
\int_{S} f(\boldsymbol{x}) \mathrm{d} \boldsymbol{x}=\alpha \widehat{Q}_{2}^{S}[f]+(1-\alpha) Q_{2}^{m i d}[f]+E_{\alpha}[f], \quad \alpha \in \mathbb{R} .
$$

where

$$
E_{\alpha}[f]=\alpha R\left(2, \frac{1}{3}\right)[f]+(1-\alpha) E[f] .
$$


Since, for all $\alpha \in \mathbb{R}, E_{\alpha}[f]=0$ whenever $f$ is a polynomial in 2 variables of total degree at least 2, the quadrature formula (4.7) has at least degree of exactness 2 .

Theorem 4.1. Let $f: S \subset \mathbb{R}^{2} \rightarrow \mathbb{R}$ be a 3-times continuous differentiable function on $S$. Then, the quadrature formula

$$
\int_{S} f(\boldsymbol{x}) d \boldsymbol{x}=M Q_{3}^{S}[f]+E_{\frac{4}{5}}[f]
$$

with

$$
M Q_{3}^{S}[f]=\frac{A(S)}{20}\left(\sum_{l=0}^{2} f\left(\frac{\boldsymbol{g}_{l}+2 \boldsymbol{v}_{l}}{3}\right)+\frac{1}{3} \sum_{l=1}^{3} f\left(\boldsymbol{g}_{l}\right)\right)
$$

has degree of exactness 3 .

Proof. Equality (4.10) follows by setting $\alpha=\frac{4}{5}$ in (4.7) and by rearranging. To prove the degree of exactness of the formula (4.9), it is sufficient to follow the same arguments used in the proof of Theorem 3.5 for $d=2$.

Finally, to obtain a quadrature formula over $S$ with degree of exactness 4 , we consider the convex combination of the quadrature formulas (4.4) and $(4.10)$

$$
\int_{S} f(\boldsymbol{x}) \mathrm{d} \boldsymbol{x}=\alpha \tilde{Q}_{3}^{S}[f]+(1-\alpha) M Q_{3}^{S}[f]+E_{\alpha}^{\prime}[f], \quad \alpha \in \mathbb{R}
$$

where

$$
E_{\alpha}^{\prime}[f]=\alpha \tilde{R}\left(\frac{9}{5}\right)[f]+(1-\alpha) E_{\frac{4}{5}}[f],
$$

with $\tilde{R}\left(\frac{9}{5}\right)[f]$ given by the Eq. (3.21) and $E_{\frac{4}{5}}[f]$ by the equation (4.8). Since, for all $\alpha \in \mathbb{R}, E_{\alpha}^{\prime}[f]=0$ whenever $f$ is a polynomial in 2 variables of total degree at least 3 , the quadrature formula (4.11) has at least degree of exactness 3 .

Theorem 4.2. Let $f: S \subset \mathbb{R}^{2} \rightarrow \mathbb{R}$ be a 3-times continuous differentiable function on $S$ and $\boldsymbol{x}^{*}$ the center of gravity of $S$. Then, the quadrature formula

$$
\int_{S} f(\boldsymbol{x}) d \boldsymbol{x}=M Q_{4}^{S}[f]+E_{\frac{1}{3}}^{\prime}[f]
$$

where

$$
M Q_{4}^{S}[f]=\frac{A(S)}{120}\left(\sum_{l=0}^{2} f\left(\boldsymbol{v}_{l}\right)+4 \sum_{l=0}^{2} f\left(\boldsymbol{g}_{l}\right)+12 f\left(\frac{\boldsymbol{g}_{l}+2 \boldsymbol{v}_{l}}{3}\right)+9 f\left(\boldsymbol{x}^{*}\right)\right)
$$

and

$$
E_{\frac{1}{3}}^{\prime}[f]=\frac{1}{3} \tilde{R}\left(\frac{9}{5}\right)[f]+\left(1-\frac{1}{3}\right) E_{\frac{4}{5}}[f],
$$

has degree of exactness 4. 
Table 1. Absolute value of the remainder terms $E_{r}^{\Delta_{2}}\left[f_{i}\right]=$ $Q_{r}^{\Delta_{2}}\left[f_{i}\right]-\int_{\Delta_{2}} f_{i}(\boldsymbol{x}) \mathrm{d} \boldsymbol{x}, i=1, \ldots, 4 ; r=1, \ldots, 10$

\begin{tabular}{lllll}
\hline$r$ & $\left|E_{r}^{\Delta_{2}}\left[f_{1}\right]\right|$ & $\left|E_{r}^{\Delta_{2}}\left[f_{2}\right]\right|$ & $\left|E_{r}^{\Delta_{2}}\left[f_{3}\right]\right|$ & $\left|E_{r}^{\Delta_{2}}\left[f_{4}\right]\right|$ \\
\hline 1 & $2.67 \mathrm{e}-3$ & $4.39 \mathrm{e}-2$ & $3.02 \mathrm{e}-4$ & $1.91 \mathrm{e}-4$ \\
2 & $1.06 \mathrm{e}-3$ & $1.03 \mathrm{e}-2$ & $3.54 \mathrm{e}-5$ & $3.87 \mathrm{e}-5$ \\
3 & $7.03 \mathrm{e}-5$ & $1.86 \mathrm{e}-2$ & $2.55 \mathrm{e}-6$ & $1.68 \mathrm{e}-6$ \\
4 & $1.73 \mathrm{e}-5$ & $4.35 \mathrm{e}-3$ & $1.75 \mathrm{e}-7$ & $1.93 \mathrm{e}-7$ \\
5 & $1.18 \mathrm{e}-6$ & $5.51 \mathrm{e}-3$ & $1.36 \mathrm{e}-8$ & $8.93 \mathrm{e}-9$ \\
6 & $2.15 \mathrm{e}-7$ & $4.01 \mathrm{e}-3$ & $6.76 \mathrm{e}-10$ & $7.55 \mathrm{e}-10$ \\
7 & $1.35 \mathrm{e}-8$ & $6.67 \mathrm{e}-4$ & $5.11 \mathrm{e}-11$ & $3.30 \mathrm{e}-11$ \\
8 & $2.60 \mathrm{e}-9$ & $1.85 \mathrm{e}-3$ & $2.02 \mathrm{e}-12$ & $2.28 \mathrm{e}-12$ \\
9 & $6.48 \mathrm{e}-10$ & $3.82 \mathrm{e}-4$ & $1.43 \mathrm{e}-13$ & $9.02 \mathrm{e}-14$ \\
10 & $5.07 \mathrm{e}-10$ & $5.86 \mathrm{e}-4$ & $5.16 \mathrm{e}-15$ & $4.94 \mathrm{e}-15$
\end{tabular}

Table 2. Absolute value of the remainder terms $\tilde{E}_{2}^{\Delta_{2}}\left[f_{i}\right]$, $\tilde{E}_{3}^{\Delta_{2}}\left[f_{i}\right], \tilde{E}_{M Q_{3}}^{\Delta_{2}}\left[f_{i}\right], \tilde{E}_{M Q_{4}}^{\Delta_{2}}\left[f_{i}\right] i=1, \ldots, 4$

\begin{tabular}{lllll}
\hline$f_{i}$ & $\left|\tilde{E}_{2}^{\Delta_{2}}\left[f_{i}\right]\right|$ & $\left|\tilde{E}_{3}^{\Delta_{2}}\left[f_{i}\right]\right|$ & $\left|\tilde{E}_{M Q_{3}}^{\Delta_{2}}\left[f_{i}\right]\right|$ & $\left|\tilde{E}_{M Q_{4}}^{\Delta_{2}}\left[f_{i}\right]\right|$ \\
\hline$f 1$ & $1.55 \mathrm{e}-3$ & $2.86 \mathrm{e}-4$ & $1.46 \mathrm{e}-4$ & $1.12 \mathrm{e}-6$ \\
$f 2$ & $3.85 \mathrm{e}-3$ & $8.64 \mathrm{e}-3$ & $4.59 \mathrm{e}-3$ & $1.83 \mathrm{e}-4$ \\
$f 3$ & $3.71 \mathrm{e}-4$ & $1.01 \mathrm{e}-5$ & $5.17 \mathrm{e}-6$ & $7.89 \mathrm{e}-8$ \\
$f 4$ & $3.76 \mathrm{e}-4$ & $1.08 \mathrm{e}-5$ & $5.51 \mathrm{e}-6$ & $7.91 \mathrm{e}-8$ \\
\hline
\end{tabular}

Proof. Equality (4.13) follows by setting $\alpha=\frac{1}{3}$ in (4.11). To prove the degree of exactness of the formula (4.12), we proceed by verifying the exactness of the quadrature formula for the monomials $x^{4}, x^{3} y, x y^{3}, x^{2} y^{2}, y^{4}$, similarly to the proof of Theorem 3.5 for $d=2$.

\section{Numerical Results in $d=2$}

To test the approximation accuracies of the proposed formulas, we consider the case $d=2$ and the standard triangle $S=\Delta_{2}$ of vertices $\boldsymbol{v}_{0}=(0,0), \boldsymbol{v}_{1}=$ $(1,0), \boldsymbol{v}_{2}=(0,1)$. The numerical experiments are conducted by considering the following set of test functions [1]

$$
\begin{aligned}
& f_{1}(x, y)=\cos \left(\sqrt{1+x^{2}+y^{2}}\right), \\
& f_{2}(x, y)=\exp \left(-\frac{(3 x-2)^{2}+(3 y-2)^{2}}{4}\right), \\
& f_{3}(x, y)=\sin \left(\frac{\pi}{4} x+\frac{\pi}{6} y\right) \\
& f_{4}(x, y)=\sinh \left(\frac{\pi}{4} x+\frac{\pi}{6} y\right) .
\end{aligned}
$$


In all the experiments, the exact value of the integrals for functions $f_{1}$ and $f_{2}$ are computed by assuming as exact the numerical integration performed by Mathematica. In Table 1, we report the absolute value of the remainder terms $E_{r}^{\Delta_{2}}\left[f_{i}\right]=Q_{r}^{\Delta_{2}}\left[f_{i}\right]-\int_{\Delta_{2}} f_{i}(\boldsymbol{x}) \mathrm{d} \boldsymbol{x}, i=1, \ldots, 4, r=1, \ldots, 10$, and, in Table 2, we display the absolute value of the remainder terms

$$
\begin{aligned}
& \tilde{E}_{2}^{\Delta_{2}}\left[f_{i}\right]=\tilde{Q}_{2}^{\Delta_{2}}\left[f_{i}\right]-\int_{\Delta_{2}} f_{i}(\boldsymbol{x}) \mathrm{d} \boldsymbol{x}, \quad i=1, \ldots, 4, \\
& \tilde{E}_{3}^{\Delta_{2}}\left[f_{i}\right]=\tilde{Q}_{3}^{\Delta_{2}}\left[f_{i}\right]-\int_{\Delta_{2}} f_{i}(\boldsymbol{x}) \mathrm{d} \boldsymbol{x}, \quad i=1, \ldots, 4, \\
& \tilde{E}_{M Q_{3}}^{\Delta_{2}}\left[f_{i}\right]=M Q_{3}^{\Delta_{2}}\left[f_{i}\right]-\int_{\Delta_{2}} f_{i}(\boldsymbol{x}) \mathrm{d} \boldsymbol{x}, \quad i=1, \ldots, 4, \\
& \tilde{E}_{M Q_{4}}^{\Delta_{2}}\left[f_{i}\right]=M Q_{4}^{\Delta_{2}}\left[f_{i}\right]-\int_{\Delta_{2}} f_{i}(\boldsymbol{x}) \mathrm{d} \boldsymbol{x}, \quad i=1, \ldots, 4 .
\end{aligned}
$$

Open Access. This article is licensed under a Creative Commons Attribution 4.0 International License, which permits use, sharing, adaptation, distribution and reproduction in any medium or format, as long as you give appropriate credit to the original author(s) and the source, provide a link to the Creative Commons licence, and indicate if changes were made. The images or other third party material in this article are included in the article's Creative Commons licence, unless indicated otherwise in a credit line to the material. If material is not included in the article's Creative Commons licence and your intended use is not permitted by statutory regulation or exceeds the permitted use, you will need to obtain permission directly from the copyright holder. To view a copy of this licence, visit http:// creativecommons.org/licenses/by/4.0/.

Publisher's Note Springer Nature remains neutral with regard to jurisdictional claims in published maps and institutional affiliations.

\section{References}

[1] Costabile, F.A., Dell'Accio, F.: New embedded boundary-type quadrature formulas for the simplex. Numer. Algorithms 45(1-4), 253-267 (2007)

[2] Costabile, F.A., Dell'Accio, F., Guzzardi, L.: New bivariate polynomial expansion with boundary data on the simplex. Calcolo 45(3), 177-192 (2008)

[3] Dunavant, D.A.: High degree efficient symmetrical Gaussian quadrature rules for the triangle. Int. J. Numer. Methods Eng. 21(6), 1129-1148 (1985)

[4] Grundmann, A., Möller, H.M.: Invariant integration formulas for the n-simplex by combinatorial methods. SIAM J. Numer. Anal. 15(2), 282-290 (1978)

[5] Guenther, R.B., Roetman, E.L.: Newton-Cotes formulae in $n$-dimension. Numer. Math. 14, 330-345 (1970)

[6] Guessab, A., Nouisser, O., Schmeisser, G.: Multivariate approximation by a combination of modified Taylor polynomials. J. Comput. Appl. Math. 196(1), 162-179 (2006)

[7] Guessab, A., Schmeisser, G.: Convexity results and sharp error estimates in approximate multivariate integration. Math. Comput. 73, 1365-1384 (2003) 
[8] Guessab, A., Nouisser, O., Schmeisser, G.: Enhancement of the algebraic precision of a linear operator and consequences under positivity. Positivity 13(4), 693-707 (2009)

[9] He, T.X.: Dimensionality Reducing Expansion of Multivariate Integration, pp. 1-23. Birkhäuser, Boston (2001)

[10] Hesthaven, J.S.: From electrostatics to almost optimal nodal sets for polynomial interpolation in a simplex. SIAM J. Numer. Anal. 35(2), 655-676 (1998)

[11] Konerth, N.: Exact multivariate integration on simplices: an explanation of the Lasserre-Avrachenkov theorem. Technical report no. 2014-05 (AU-CasMathstats) (2014)

[12] Lyness, J.N., Cools, R.: A survey of numerical cubature over triangles. In: Proceedings of Symposia in Applied Mathematics, vol. 48. American Mathematical Society, pp. 127-150 (1994)

[13] Shunn, L., Ham, F.: Symmetric quadrature rules for tetrahedra based on a cubic close-packed lattice arrangement. J. Comput. Appl. Math. 236(17), 43484364 (2012)

[14] Silvester, P.: Symmetric quadrature formulae for simplexes. Math. Comput. 24(109), 95-100 (1970)

[15] Vermolen, F.J., Segal, A.: On an integration rule for products of barycentric coordinates over simplexes in $\mathbb{R}^{n}$. J. Comput. Appl. Math. 330, 289-294 (2018)

[16] Wandzurat, S., Xiao, H.: Symmetric quadrature rules on a triangle. Comput. Math. Appl. 45(12), 1829-1840 (2003)

[17] Zhang, L., Cui, T., Liu, H.: A set of symmetric quadrature rules on triangles and tetrahedra. J. Comput. Math. 27, 89-96 (2009)

Filomena Di Tommaso

Department of Mathematics and Computer Science

University of Calabria

Via P. Bucci, cubo 30A

87036 Rende

Italy

e-mail: filomena.ditommaso@unical.it

Benaissa Zerroudi

University Ibn Tofail

Kenitra

Morocco

e-mail: zerroudi@gmail.com

Received: September 25, 2019.

Revised: March 11, 2020.

Accepted: August 2, 2020. 